In Dispraise of Inconsistent DNA Relative Frequency Estimates

by

Seymour Geisser

University of Minnesota

Technical Report No. 620

March 1997 


\title{
In Dispraise of Inconsistent DNA Relative Frequency Estimates
}

\author{
Seymour Geisser*
}

\section{Introduction}

A series of papers ${ }^{1,2,3,4}$ criticized some of the statistical methods employed and the databases gathered by two of the principal forensic laboratories, the FBI and Cellmark, that are engaged in DNA matching and profiling. The major criticisms were: (a) lack of random sampling and adequate sample size, e.g. the fact that Cellmark's small Black database was obtained from rare blood donor volunteers at a Detroit blood bank and similarly their Caucasian database from the Blood Bank of Delaware; (b) lack of mutual independence among alleles within probes and between probes; (c) the fact that the product rule, under the best of assumptions, i.e. random sampling and mutual independence, will underestimate the relative frequency of a profile; (d) the lack of blind, external and periodic proficiency tests; and (e) the fact that the FBI $5 \%$ window for the original match led to a theoretically computable false exclusion rate for 4 probes of between $10^{-8}$ and $10^{-10}$ without any notion of what the false inclusion rate would be and claiming it to be a conservative procedure when augmented by the FBI bins $^{5}$. Failure on the part of the NRC report to criticize forensic laboratories for these flaws is inexcusable.

"Seymour Geisser is Professor and Director of the School of Statistics, University of Minnesota, Minneapolis.

Work was supported in part by NIGMS grant 25271.

${ }^{1}$ Seymour Geisser, Some Statistical Issues in Forensic DNA, in MODELLING AND PREDICTION 3, Springer (Jack C. Lee, et al. eds., 1996).

${ }^{2}$ Seymour Geisser \& Wesley O. Johnson, Testing Hardy-Weinberg Equilibrium on Allelic Data from VNTR Loci, 51 AMERICAN JOURNAL OF HUMAN GENETICS 1084 (1992).

${ }^{3}$ Seymour Geisser \& Wesley O. Johnson, Testing Independence of Fragment Lengths within VNTR Loci, 53 AMERICAN JOURNAL OF HUMAN GENETICS 1103 (1993).

${ }^{4}$ Seymour Geisser \& Wesley O. Johnson, Testing Independence when the Form of the Bivariate Distribution is Unspecified, 14 STATISTICS IN MEDICINE 1621 (1995).

${ }^{5}$ Bruce Budowle et al., A Preliminary Report on Binned General Population Data on Six VNTR Loci in Caucasians, Blacks and Hispanics from the United States, 18 CRIME LIBRARY DIGEST 9 (1991). 
In what follows we take up another flawed feature of the FBI procedure and an inadequate feature of the databases that Cellmark has been using.

\section{Inconsistency of Initial Match and Database Match}

The FBI's initial match window, which is essentially $\pm 5 \%$ of the band value is then augmented by their binning procedure which initially had 31 bins. This was criticized by Fung ${ }^{6}$ who demonstrated that about more than half of these bins were less than $\pm 5 \%$ of their midpoint in size, or of width $10 \%$, belying the claim of conservatism.

However, because of the distribution of bands and size of the database, the FBI has some time ago adjusted their binning procedure to between 13 and 24 bins depending on the probe used.

A second critical issue, not addressed by Fung, is that the initial match is based on an intragel comparison while the match between the suspect profile and those in the database is necessarily an intergel comparison. For bands less than 10,000 basepairs, the intergel standard deviation is approximately 1.82 times the intragel standard deviation. This would indicate that, for the sake of consistency, a match of suspect band to a database band should be increased to $\pm 9.1 \%$ of the band value. In Table 1 , we investigate the size $\%$ of the bins for the Caucasian database, the largest of the several FBI databases, in terms of the number and percent of bins greater than $\pm 9.1 \%$ of midpoint band size (columns 2 and 3 ) and the fraction of the database residing in bins greater than this $18.2 \%$ width, column 4 . From there, it is clear that very few bins are greater than this standard in width and the percentage of individuals in those bins is quite small, both in magnitude and in relation to proportion of bins below $18.2 \%$. Even if all other assumptions such as random sampling, mutual independence of alleles within and between probes are considered to be correct, and a correction made for underestimation by the product rule, the FBI's claim to conservatism based on a consistent relationship between their initial match window and their binning procedure is clearly false.

It is also interesting to note the difference between the percentages in the last two columns

\footnotetext{
${ }^{6}$ Wing K. Fung, $10 \%$ or $5 \%$ Match Window in DNA Profiling, 78 FORENSIC SCI. INT. 111.
} 
which reflect mainly end effects, i.e. the first and last bins for each probe (essentially the tails of the distribution) which are generally very large relative to the number in the bin. The last bin of course is infinite in size and hence always larger than $18.2 \%$. If these were eliminated, noting that the fraction of the individuals in the database is very small in these bins, the situation would further diminish the claim of conservatism, which was based on criteria inconsistently applied. If anything, it demonstrates that the FBI's estimates would appreciably underestimate the relative frequency of profiles without even considering several other flawed assumptions.

\section{Pairwise Comparisons}

To have an idea of how much underestimation there might be, we can calculate in a database the number of matches involving all possible pairs of individual profiles. We shall use the FBI Caucasian database which is the largest of their racial databases to provide us with some idea of the extent of the underestimation. The five most commonly used RFLP probes will be investigated. A sixth D14S13 is less frequently used in court cases.

Two different estimates are compared in Table 2, the pairwise direct count using a $\pm 9.1 \%$ floating bin which does not depend on independence and the FBI adjusted binning procedure giving the 5, 25, 50, 75 and 95 percentile values. The relative frequency for the direct count varies in the range of 1 in 4,700-53,000 for 3 probe matches. For 4 probes it varies in the range of 1 in 70,000-80,000. We also note that there was one 5-probe match out of the 73,000 odd comparisons and that match turned out to be a six-probe match if D14S13 were included! These values are quite different from what using the product rule with the FBI bins yield and certainly different from the most frequently reported values of 1 in many millions and billions in court cases. The NRC report also quotes a similar study done by Risch and Devlin ${ }^{7}$ on pairs using the direct count. They reported no matches in 4 or 5 probes among 7.6 million pairs of profiles. But they used a $\pm 2.4 \%$ window so these results were quite inconsistent with even the FBI initial $\pm 5 \%$ window. The NRC report also stated that

${ }^{7}$ Neil J. Risch \& Bernard Devlin, On the Probability of Matching DNA Fingerprints, 255 SCIENCE 717 (1992). 
out of 58 million pairwise comparisons, only 2 matches were found on the basis of a personal communication from $\mathrm{R}$. Chakraborty. No mention is made of the match criterion used. While we agree with the NRC report that the same criterion (a floating bin) should be used for the initial match and the database match-we assert that it should be adjusted for the difference in variation inherent in an intragel and intergel comparison. It is clear from Table 2 that the inconsistent FBI procedure from a 4 probe match could easily underestimate the relative frequency in the range of $10^{3}$ to $10^{5}$ or more. Calling such a procedure conservative or as favoring the defendant defies common sense.

We also point out that with regard to databases that were used by Cellmark, not only are the totals inadequate, see Table 3 , but the number of individuals that have measurements on all 5 of their probes are 2 for Blacks, 59 for Hispanics, and 75 for Caucasians. For the inadequacy of individuals measured on 4 probes out of 5 probes, see Table 4. 
Table 1: FBI Caucasian Database (Rebinned)
(1)
(2) (3)
(4)

No. of bins No. $> \pm 9.1 \% \quad \% \quad \%$ of database in (2)

$\begin{array}{ccccc}\text { D2S44 } & 21 & 3 & 14.3 & 2.1 \\ \text { D17S79 } & 13 & 3 & 23.1 & 2.0 \\ \text { D1S7 } & 26 & 2 & 7.7 & 5.5 \\ \text { D4S139 } & 17 & 2 & 11.8 & 10.6 \\ \text { D10S28 } & 23 & 2 & 8.7 & 2.1 \\ \text { D14S13 } & 24 & 5 & 20.8 & 2.6\end{array}$


Table 2: Relative frequency in FBI Caucasian database for 3 and 4 probes using a $\pm 9.1 \%$ window, pairwise direct count, and FBI bins using product rule.

\section{Relative frequency (1 out of)}

\begin{tabular}{|c|c|c|c|c|c|c|c|}
\hline \multirow[t]{2}{*}{$\begin{array}{l}3 \text { Probe Matches } \\
\text { (Omitted Probes) }\end{array}$} & \multirow[t]{2}{*}{$\begin{array}{c}\text { No. of } \\
\text { Individuals }\end{array}$} & \multirow{2}{*}{$\begin{array}{c}\begin{array}{c}\text { Pairwise } \\
\text { Direct Count }\end{array} \\
\pm 9.1 \%\end{array}$} & \multicolumn{5}{|c|}{$\begin{array}{l}\text { Product Rule } \\
\text { Percentiles using FBI Bins }\end{array}$} \\
\hline & & & $95 \%$ & $75 \%$ & $50 \%$ & $25 \%$ & $5 \%$ \\
\hline D10S28, D17S79 & 565 & $5.3 \times 10^{4}$ & $1.8 \times 10^{5}$ & $5.9 \times 10^{5}$ & $1.7 \times 10^{6}$ & $5.1 \times 10^{6}$ & $2.6 \times 10^{7}$ \\
\hline D17S79, D4S139 & 410 & $4.2 \times 10^{4}$ & $6.3 \times 10^{5}$ & $1.7 \times 10^{6}$ & $4.9 \times 10^{6}$ & $1.2 \times 10^{7}$ & $5.2 \times 10^{7}$ \\
\hline D4S139, D10S28 & 548 & $1.0 \times 10^{4}$ & $5.3 \times 10^{4}$ & $1.3 \times 10^{5}$ & $3.9 \times 10^{5}$ & $1.4 \times 10^{6}$ & $1.0 \times 10^{7}$ \\
\hline D1S7, D17S79 & 405 & $4.1 \times 10^{4}$ & $1.8 \times 10^{5}$ & $5.5 \times 10^{5}$ & $1.7 \times 10^{6}$ & $4.9 \times 10^{6}$ & $2.5 \times 10^{7}$ \\
\hline D10S28, D1S7 & 549 & $4.7 \times 10^{3}$ & $1.5 \times 10^{4}$ & $4.6 \times 10^{4}$ & $1.2 \times 10^{5}$ & $4.5 \times 10^{5}$ & $5.8 \times 10^{6}$ \\
\hline D4S139, D1S7 & 401 & $5.7 \times 10^{3}$ & $5.4 \times 10^{4}$ & $1.2 \times 10^{5}$ & $3.0 \times 10^{5}$ & $1.2 \times 10^{6}$ & $9.7 \times 10^{6}$ \\
\hline D2S44, D17S79 & 402 & $4.0 \times 10^{4}$ & $3.2 \times 10^{5}$ & $9.8 \times 10^{5}$ & $2.9 \times 10^{6}$ & $8.3 \times 10^{6}$ & $3.8 \times 10^{7}$ \\
\hline D2S44, D10S28 & 543 & $1.1 \times 10^{4}$ & $2.7 \times 10^{4}$ & $7.4 \times 10^{4}$ & $2.0 \times 10^{5}$ & $9.2 \times 10^{5}$ & $9.0 \times 10^{6}$ \\
\hline D2S44, D4S139 & 396 & $1.3 \times 10^{4}$ & $9.2 \times 10^{4}$ & $2.1 \times 10^{5}$ & $5.4 \times 10^{5}$ & $2.0 \times 10^{6}$ & $1.6 \times 10^{7}$ \\
\hline D2S44, D1S7 & 397 & $7.9 \times 10^{3}$ & $2.6 \times 10^{4}$ & $7.0 \times 10^{4}$ & $1.8 \times 10^{5}$ & $8.9 \times 10^{5}$ & $6.1 \times 10^{6}$ \\
\hline
\end{tabular}

\section{Probe Matches}

(Probe Omitted)

D17S79
D10S28
D4S139
D1S7
D2S44

$\begin{array}{lllllll}396 & 7.8 \times 10^{4} & 2.4 \times 10^{7} & 1.1 \times 10^{8} & 3.6 \times 10^{8} & 1.1 \times 10^{9} & 6.3 \times 10^{9} \\ 535 & 7.1 \times 10^{4} & 2.1 \times 10^{6} & 8.8 \times 10^{6} & 2.7 \times 10^{7} & 1.1 \times 10^{8} & 1.6 \times 10^{9} \\ 391 & 7.6 \times 10^{4} & 6.7 \times 10^{6} & 2.4 \times 10^{7} & 7.5 \times 10^{7} & 2.9 \times 10^{8} & 2.1 \times 10^{9} \\ 391 & 7.6 \times 10^{4} & 2.0 \times 10^{6} & 7.5 \times 10^{6} & 2.5 \times 10^{7} & 9.7 \times 10^{7} & 1.3 \times 10^{9} \\ 387 & 7.5 \times 10^{4} & 3.7 \times 10^{6} & 1.3 \times 10^{7} & 4.0 \times 10^{7} & 2.2 \times 10^{8} & 1.9 \times 10^{7}\end{array}$


Table 3: Number of individuals with values on Cellmark probes

\begin{tabular}{c|rrr} 
Total on each Probe & Blacks & Caucasians & Hispanics \\
\hline MS1 & 240 & 262 & 215 \\
MS31 & 238 & 264 & 183 \\
MS43 & 223 & 294 & 192 \\
G3 & 200 & 325 & 168 \\
YNH24 & 146 & 208 & 110
\end{tabular}

Table 4: Number of individuals with values on 4 probes

\begin{tabular}{c|rrr} 
Omitted Probe & Blacks & Caucasians & Hispanics \\
\hline MS1 & 2 & 79 & 63 \\
MS31 & 2 & 108 & 73 \\
MS43 & 2 & 77 & 72 \\
G3 & 31 & 91 & 76 \\
YNH24 & 8 & 153 & 109
\end{tabular}

